\title{
Microcracks in subchondral bone plate is linked to less cartilage damage
}

Zarka $\mathbf{M}^{1}$, Hay $\mathbf{E}^{1}$, Ostertag $\mathbf{A}^{1}$, Marty $\mathbf{C}^{1}$, Chappard $\mathbf{C}^{2}$, Oudet $\mathrm{F}^{3}$, Engelke $\mathrm{K}^{4}$, Laredo $J^{2-5}$, Cohen-Solal M1

${ }^{1}$ Inserm UMR1132 and Paris Diderot University, Paris France

${ }^{2}$ B2OA, UMR CNRS7052, Paris Diderot University, Paris, France,

${ }^{3}$ Direction à la recherche, Sorbonne Universités, Université de technologie de Compiègne, Compiègne, France

${ }^{4}$ Institute of Medical Physics, University of Erlangen, Erlangen, Germany; Dept of Medicine, University Hospital Erlangen, Germany

${ }^{5}$ Department of Bone and Joint imaging, Hôpital Lariboisière, Paris, France

mylene.zarka@inserm.fr eric.hay@inserm.fr christine.chappard@inserm.fr agnes.ostertag@inserm.fr caroline.marty@inserm.fr francois.oudet@utc.fr klaus.engelke@imp.uni-erlangen.de jean-denis.laredo@lrb.aphp.fr

Corresponding author: Martine Cohen-Solal Inserm U1132 and university Paris-Diderot Hopital Lariboisiere 2 rue Ambroise Paré, 75010 Paris martine.cohen-solal@inserm.fr 


\section{Abstract}

Objectives: Osteoarthritis (OA) is a disease of the whole joint characterized by cartilage loss and subchondral bone remodeling. The role of microcracks in cartilage integrity and subchondral bone homeostasis is not fully understood. The main goal of this work was to evaluate microcrack density in both calcified cartilage and subchondral bone plate in relation to cartilage damage in humans and to better define the association of microcracks and osteocyte density in subchondral bone.

Methods: We investigated 18 bone cores from cadaveric human knees that were stained with En-Bloc Basic Fuchsin. We quantified microcrack density, osteocyte density, cartilage surfaces and cartilage damage. The presence of microcracks was confirmed for each bone core by scanning electron microscopy. Finally, trabecular subchondral bone parameters were measured by micro-CT.

Results: Microcracks were detected in both calcified cartilage and subchondral bone plate. The density of microcracks in both calcified cartilage (CC) and subchondral bone plate (SBP) was negatively correlated with cartilage damage $(\mathrm{r}=-0.44, \mathrm{p}<0.05)$. The presence of microcracks in SBP was associated with a lower histological OA score. Osteocytes formed a dendrite network that abruptly stopped at the border of calcified cartilage. Osteocyte density in subchondral bone plate was increased in the presence of microcracks in calcified cartilage.

Conclusions: Subchondral bone plate microcracks might be required for maintaining cartilage homeostasis. Microcracks in calcified cartilage may trigger osteocyte density in subchondral bone plate with subsequent regulation of subchondral bone remodeling to prevent cartilage damage. 


\section{Highlights}

- Subchondral bone contains a high density of osteocytes, the dendrites of which are bordering the calcified cartilage.

- Microcracks are negatively correlated with cartilage damage, suggesting that they might be required for maintaining cartilage homeostasis.

- Microcracks in the calcified cartilage are associated with increased osteocyte density in the subchondral bone

- Microcracks in the calcified cartilage might regulate subchondral bone remodeling

Keywords: osteoarthritis, bone, microcracks, osteocytes, mechanical loading 


\section{Introduction}

Bone microdamage, a physiological process induced by bone strain, was first described by Frost, in 1960 [1]. Microcracks can occur at different location notably in subchondral bone and calcified cartilage $[2,3]$. Bone microcracks were found to promote bone remodeling and were associated with impaired bone mechanical properties that might contribute to fatigue damage and further expose to fractures [4-7].

The density of microcracks increases with age as shown for the first time by Schaffler and colleagues, and they are mostly observed in older women [8-11]. Several studies have demonstrated an association of microcrack density with increased bone resorption induced by osteocyte apoptosis near bone microdamage [12-14]. Therefore, osteocytes, the cells embedded in the bone matrix, play a pivotal role in the initiation of bone remodeling $[12,15,16]$. Osteocyte density in the trabecular and the cortical bone is negatively correlated with microcrack number and aging $[9,10,17]$. Hence, the age-related decrease in osteocyte number might be associated to a low ability to induce bone remodeling as a response of bone to microdamage.

Osteoarthritis (OA) is a worldwide joint disease characterized by cartilage loss and subchondral changes. Subchondral bone is involved in the initiation and progression of OA [18-21]. The crosstalk between bone and cartilage includes diffusion of small molecules within the joint via the subchondral bone, or driven by microcracks that facilitate the circulation of growth factors and cytokines [22]. Moreover, high subchondral bone remodeling is associated with increased osteoclastogenesis, whose inhibition protects against cartilage lesions [19,23,24]. This mechanism involves cytokines such as RANKL/osteoprotegerin (RANKL/OPG) produced by bone cells preventing OA progression [23]. In addition, mechanical loading induces $\mathrm{Wnt} / \beta$-catenin signaling in osteocytes and the secretion of soluble factors by osteoblast/osteocytes that can regulate cartilage metabolism 
[25-28]. Indeed, loss of sclerostin mostly expressed by osteocytes promotes OA in mice, suggesting that bone cells may prevent cartilage degradation [29]. These findings highlight the role of subchondral bone in regulating cartilage degradation during OA.

In the context of OA, a low number of trabecular microfractures and a high density of osteocyte lacunae at the subchondral bone plate have been reported [30,31]. Moreover, others reported an increased in microcrack density at both subchondral bone plate and subchondral trabeculae in OA patients with bone-marrow lesions [32]. This suggests that microcracks in the subchondral bone may be involved in maintaining cartilage integrity perhaps via bone remodeling.

In trabecular bone as well in subchondral bone, microcracks could be related to two distinct mechanisms. Microcracks may indicate a failure of bone to support mechanical loading and promote the repair of bone matrix in one hand, or also could be a protective factor against greater bone damage in another hand. Microcracks can occur in SBP and in calcified cartilage (CC), thereby providing a communication path for cartilage-bone crosstalk. Calcified structures close to the articular cartilage include $\mathrm{CC}, \mathrm{SBP}$ and trabecular bone, each of which could help provide soluble factors able to influence cartilage homeostasis.

The aim of this study was to proceed to a descriptive evaluation of microcrack density in both $\mathrm{CC}$ and SBP in relation to cartilage damage in humans and to better define the association of microcracks with osteocyte density in subchondral bone. 


\section{Materials and Methods}

\subsection{Human biopsy}

We included 18 cadaveric human knees obtained from individuals who had bequeathed their bodies to science. The cadavers were obtained from the Saint-Pères Laboratory of Pathology, University Paris VI, France. The cadavers represented 11 women and 7 men (mean [SD] age 82.2 [9.6] years at death [range 63-101]). Medical history and OA status were not available for any of the subjects. Knee specimens were kept frozen at $-20^{\circ} \mathrm{C}$ until analysis, then thawed at room temperature, and superficial tissues were removed to expose joint surfaces. For this study, one bone core per knee specimens were taken from the medial tibia plateau by use of a trephine with $7 \mathrm{~mm}$ internal diameter (BROT, Argenteuil, France) under constant water irrigation. Bone biopsies used in the present study were always taken at the same site in the knee (Supplemental Figure 1A). Collection of the samples used in this study was previously described [33].

\subsection{Image acquisition by micro-CT}

Micro-CT acquisition from bone cores involved use of a SkyScan 1172 Scanner (Bruker, Coventry, UK). Samples were placed vertically and scanned with the following settings: aluminum and copper filter, $0.5 \mathrm{~mm}$; resolution, $9 \mu \mathrm{m}$; energy, $69 \mathrm{kV}$; intensity, $100 \mu \mathrm{A}$; and integration time, $380 \mathrm{~ms}$. 3-D reconstruction and analysis involved use of DataViewer and CTanalyser. Bone microarchitecture was analyzed on the total bone cores (Supplemental Figure 1B). Subchondral trabecular bone underneath the SBP is defined from cross section of bone, as the beginning of subchondral bone where trabecular bone appears. Subchondral bone parameters was defined underneath the SBP including a region of interest of $1.79 \mathrm{~mm}$. Parameters measured were bone volume/tissue volume $(\%)$, trabecular thickness $(\mu \mathrm{m})$, trabecular separation $(\mu \mathrm{m})$, and trabecular number $(1 / \mathrm{mm})$. 


\subsection{Histology}

The 18 bone cores were analyzed by histology. To measure microcracks and avoid section artefacts, specimens were stained by the En-Bloc Basic Fuchsin staining protocol described in the Burr \& Hooser technical note [34]. Briefly, after preservation in 70\% ethanol, specimens were stained with $1 \%$ basic fuchsin in a graded alcohol series under vacuum. Then, samples were embedded in methylmethacrylate and sectioned in $5-\mu \mathrm{m}$ thick sections by using a microtome Leica SM 2500 or $100-\mu$ m thick sections by using an Isometer.

Quantification of microcrack density, osteocyte density and cartilage surfaces involved use of the Histolab software package developed for bone histomorphometry (Microvision, Evry, France). Microcracks were measured in CC and SBP in $100-\mu \mathrm{m}$ thick sections. The criteria described by Burr \& Stafford were used to identify microcracks and to discriminate from vessels [35]. Microcrack identification is based on the following criteria: 1) intermediate in size, larger than canaliculi but smaller than vascular channels; 2) sharp borders with a halo of basic fuchsin staining; 3) stained through the depth of the section; and 4) when the depth of focus is changed, edges of cracks are more deeply stained than the intervening space. Microcracks were measured under light microscopy on one or two sections according to the quality as illustrated in Figure 1 and the number and density were quantified.

Cartilage damage was measured in 5 - $\mu \mathrm{m}$ thick sections stained with toluidine blue after previous incubation with ethanol 70 to remove fuschin staining. Safranin O staining could not be performed because of interference with methyl methacrylate. OA score was determined according to the Pritzker grading system on three sections of 5- $\mu \mathrm{m}$ thick sections [36]. Briefly, the grading system involves six grades based on key features: Grade 0, surface intact and cartilage morphology intact; Grade 1, surface intact with superficial fibrillation (abrasion); Grade 2, surface discontinuity, toluidine blue stain depletion in upper one third of 
cartilage; Grade 3, erosion, toluidine blue stain depletion in lower two thirds of cartilage; Grade 4, denudation, sclerotic bone; and Grade 5, deformation, bone remodeling.

\subsection{Environmental Scanning Electron Microscopy (ESEM)}

Environmental Scanning Electron Microscopy, ESEM, (XL-30, Philips, the Netherlands) allows the examination of samples under moderate vacuum. Samples are observed without usual delicate SEM preparation procedure. ESEM thus provide an imaging free of preparation artifacts or high vacuum effects [37,38]. Images were obtained with backscattered electrons in low-vacuum mode (1.4 torr) on non-metallized polished bone samples. The same sections of $100 \mu \mathrm{m}$ were used for each core to identify the presence of microcracks.

\subsection{Statistical analysis}

The histomorphometric parameters and Pritzker scoring data obtained from bone cores were not normally distributed (Shapiro-Wilk test). Correlation analysis of biopsy findings was achieved with Spearman or Pearson test as a function of Shapiro-Wilk test results. Group differences were assessed by Mann-Whitney and data are expressed as mean $\pm \mathrm{SD}$. $\mathrm{P}<0.05$ was considered statistically significant. The effect of microcrack distribution was determined by a two-way analysis of variance considering the two following factors: presence or absence of microcracks in the SBP, and presence or absence of microcracks in the CC. Statistical analyses involved using GraphPad Prism 7.00 (GraphPad Software, La Jolla, CA, USA). 


\section{Results}

\subsection{Microcracks are associated with low cartilage damage}

Microcracks were measured by fuschin staining, which allows for discriminating existing microcracks versus those induced by the processing of the plastic blocks. Microcracks were present in all subchondral calcified tissues, at the CC alone or SBP alone or both (Figure 1). Parameters associated with the microcracks are shown in Table I. Microcrack number in CC or SBP did not differ by age, and age and OA score were not correlated $(\mathrm{p}=0.47)$. OA score was significantly lower with than without microcracks in $\operatorname{SBP}(2.14 \pm 0.32$ vs $3.67 \pm 0.31$, $\mathrm{p}<0.01$, Table I) but was not significant for $\mathrm{CC}(2.38 \pm 0.41$ vs $3.00 \pm 0.41, \mathrm{p}=0.17)$. We found a significant negative correlation between the density of microcracks in SBP and CC and OA score $(\mathrm{r}=-0.45 \mathrm{p}=0.04$; Figure $2 \mathrm{~A})$. OA score was lower in specimens with microcracks at both CC and SBP areas than those with no microcracks (none) $(2.06 \pm 0.52 \mathrm{vs}$ $4.00 \pm 0.29, \mathrm{p}<0.05$, Figure 2B). Microcracks in SBP were associated with a lower OA score and this is independent of the presence of microcracks in $\mathrm{CC}$ (Figure 2C).

\subsection{Osteocyte density and microcracks}

In SBP, osteocytes formed an extended dendrite network, with a sharp stop when they reached the border of the CC (Figure 1). SBP osteocyte density was higher with than without microcracks in CC $(724.0 \pm 60.6$ vs $548.6 \pm 32.6, p=0.015$, Figure $3 \mathrm{~A})$ but did not depend on the presence of microcracks in SBP $(581.1 \pm 41.5$ vs $651.3 \pm 67.3, p=0.331$, Figure $3 B)$. In addition, osteocyte density in SBP was inversely correlated with trabecular subchondral bone volume $(r=-0.47 \mathrm{p}=0.04$, Figure $3 \mathrm{C})$. Finally, none of the other microstructural parameters of trabecular bone was significantly modified in the presence or not of microcracks in $\mathrm{CC}$ or SBP (Table I), and OA score was not correlated with trabecular thickness ( $\mathrm{r}=-0.18)$, trabecular 
separation $(\mathrm{r}=-0.19)$ or trabecular number $(\mathrm{r}=-0.31)$ in the different subgroups $($ all $\mathrm{p}>0.05)$ (Supplemental Figure 2).

\section{Discussion}

We focused on microcracks in CC and SBP and found that they occur separately or concomitantly in calcified structures close to cartilage. Our major finding is that the presence of microcracks is associated with less cartilage damage.

In bone fragility, microcracks were proposed to be a response to injury or overload, then stimulate osteocyte apoptosis and initiate bone remodeling and repair to maintain bone mass and strength $[12,13,16]$. In OA, microcracks might be early events of the disease as a consequence of mechanical loading to trigger bone and cartilage cell activity and maintain joint integrity. However, it is also likely that microcracks may reflect a way by which articular cartilage might be protected from trauma by means of dispersion of energy through microcracks in response to mechanical loading.

In OA mice, the progression of cartilage damage parallels the thickening of the SBP [28], which could be mediated by mechanical overload and also by diffusion of molecules from bone and CC through the microcracks [22]. Such a crosstalk includes several factors that might influence cartilage catabolism, such as RANKL/OPG [13,23,39], inhibitors of Wnt signaling [28,29] and newly discovered Pannexin-1 [40].

Most reports describing bone and cartilage crosstalk focused on subchondral trabecular bone or the SBP. Here, we found lower OA score with than without microcracks in SBP, which suggests a physiological role in the homeostasis of cartilage. It is also possible that the presence of microcracks associated with lower OA score might reflect a greater capacity to promote microcracks in an undamaged SBP than in an OA SBP that is known to be less mineralized. 
Soluble factors might be mediators of chondrocyte metabolism. Such a crosstalk is made possible by the diffusivity of molecules from bone to cartilage in OA mice and in controls [41] and therefore indicates that the effect of factors might depend on the concentration produced by activated cells. Indeed, our data suggest that soluble factors produced by bone cells might be involved in cartilage breakdown [23,29]. In addition, similar cartilage protection is achieved with the presence of microcracks in SBP. Therefore, microcracks might drive the diffusion of such factors produced by hypertrophic chondrocytes or bone cells to maintain cartilage homeostasis.

We found microcracks in CC associated with increased SBP osteocyte density, whereas SBP showed the same osteocyte density with and without microcracks. The adaptive response to loading and bone repair occurs with the emergence of microcracks and loss of osteocytes [42]. Our results could also suggest that higher mechanical loading at the SBP might be related to the failure of $\mathrm{CC}$ to absorb mechanical strains that leads to the activation of osteocytemediated bone remodeling.

Microcracks are mostly reported in trabecular bone as a result of impact loading or a failure of bone strength in osteoporotic women as low bone mineral density was reported to be associated with bone microdamage accumulation in postmenopausal women with osteoporosis [43]. In cortical bone, a structure similar to SBP, fatigue loading induces osteocyte apoptosis when close to microcracks and subsequent osteoclastic bone resorption, so osteocytes are key cells for initiating bone repair [44]. Although the level of bone remodeling is low in SBP, osteocytes might promote the diffusion of molecules such as vascular endothelial growth factor or RANKL, which induces chondrocyte hypertrophy and progression of $\mathrm{CC}$ thickening. In turn, chondrocyte-secreted RANKL might promote osteoclast recruitment in subchondral bone that would contribute to the CC microcracks [45]. 
Such a bi-directional crosstalk between chondrocytes of the CC and osteocytes of SBP would explain the circulation of local factors able to inhibit cartilage breakdown, as shown in Figure 4. Unfortunately, frozen samples did not allow for measuring osteoclastic bone resorption. Here, we did not find any difference in trabecular microarchitecture in samples with or without microcracks, or any correlations between bone parameters and presence of microcracks. Therefore, bone remodeling in trabecular bone close to the joint might be influenced by additional parameters secondary to joint loading, as previously shown in racehorses [46]. However, we observed that the higher the osteocyte density in the SBP, the lower is the underlying bones volume. This could result of an increase sclerostin production due to the high osteocytes density, affecting wnt pathway and reducing bone formation. This effect is observed at the early stage of OA onset; on later stage, as described in mice, the production of sclerostin decreases in the SBP along with high bone formation [47].

Taken together, our data show that SBP microcracks is associated with a maintained cartilage homeostasis and also that $\mathrm{CC}$ microcracks is linked to increased osteocytes density in the SBP (Figure 4). In this schematic view, we propose that lack of microcracks is associated with decreased SBP remodeling and cartilage breakdown.

In conclusion, microcracks are associated with reduced cartilage loss, which suggests a physiological role. They can occur as a response to preventing tissue degradation in the joint in part by regulating osteocyte activity in the SBP.

Acknowledgments: We thank Vincent Lethong and Jhoicy Justin Vimaladas for technical contribution.

\section{Author contributions}

Conception and design (ZM, HE, CSM); analysis and interpretation of the data (ZM, HE, $\mathrm{CSM})$; drafting of the article (ZM, CSM); critical revision of the article for important 
intellectual content (ZM, HE, CSM); final approval of the article (all authors); provision of study materials or patients (CC, LJD, CSM); statistical expertise (ZM, OA); obtaining funding (CSM); administrative, technical, or logistical support (MC, OA); collection and assembly of data (MZ, OA, CC, CSM).

\section{Role of the funding source}

The study was supported by the "foundation de l'Avenir" and the Sybil network.

\section{Conflict of interest}

Authors have no conflict of interest to disclose with the work. 


\section{References}

[1] H. Frost, Presence of microscopic cracks in vivo in bone, Henry Ford Hosp Med Bull. 8 (1960) 25-35.

[2] S. Mori, R. Harruff, D. Burr, Microcracks in articular calcified cartilage of human femoral heads, Arch Pathol Lab Med. 117 (1993) 196-8.

[3] L. Sokoloff, Microcracks in the calcified layer of articular cartilage, Arch Pathol Lab Med. 117 (1993) 191-195.

[4] D.B. Burr, M.R. Forwood, D.P. Fyhrie, R.B. Martin, M.B. Schaffler, C.H. Turner, Bone microdamage and skeletal fragility in osteoporotic and stress fractures, J. Bone Miner. Res. 12 (1997) 6-15. doi:10.1359/jbmr.1997.12.1.6.

[5] F.M. Lambers, A.R. Bouman, C.M. Rimnac, C.J. Hernandez, Microdamage caused by fatigue loading in human cancellous bone: Relationship to reductions in bone biomechanical performance, PLoS One. 8 (2013) e83662. doi:10.1371/journal.pone.0083662.

[6] G. Wang, X. Qu, Z. Yu, Changes in the mechanical properties and composition of bone during microdamage repair, PLoS One. 9 (2014) e108324. doi:10.1371/journal.pone.0108324.

[7] S. Mori, D. Burr, Increased intracortical remodeling following fatigue damage, Bone. 14 (1993) 103-109.

[8] T.L. Norman, Z. Wang, Microdamage of human cortical bone: Incidence and morphology in long bones, Bone. 20 (1997) 375-379. doi:10.1016/S87563282(97)00004-5.

[9] S. Mori, R. Harruff, W. Ambrosius, D.B. Burr, Trabecular bone volume and microdamage accumulation in the femoral heads of women with and without femoral neck fractures, Bone. 21 (1997) 521-526. doi:10.1016/S8756-3282(97)00200-7.

[10] S. Wong, R. Evans, C. Dunstan, E. Hills, The effect of age on bone composition and viability in the femoral head, J Bone Jt. Surg Am. 67 (1985) 274-283.

[11] M.B. Schaffler, K. Choi, C. Milgrom, Aging and Matrix Microdamage Accumulation in Human Compact Bone, Bone. 17 (1995) 521-525.

[12] O.D. Kennedy, D.M. Laudier, R.J. Majeska, H.B. Sun, M.B. Schaffler, Osteocyte Apoptosis is Required for Production of Osteoclastogenic Signals Following Bone Fatigue in vivo, Bone. 64 (2014) 132-137. doi:10.3174/ajnr.A1256.Functional.

[13] O.D. Kennedy, B.C. Herman, D.M. Laudier, R.J. Majeska, H.B. Sun, M.B. Schaffler, Activation of resorption in fatigue-loaded bone involves both apoptosis and active proosteoclastogenic signaling by distinct osteocyte populations, Bone. 50 (2012) 11151122. doi:10.1111/j.1743-6109.2008.01122.x.Endothelial.

[14] O. Verborgt, G.J. Gibson, M.B. Schaffler, Loss of osteocyte integrity in association with microdamage and bone remodeling after fatigue in vivo, J. Bone Miner. Res. 15 (2000) 60-67. doi:10.1359/jbmr.2000.15.1.60. 
[15] B. Herman, L. Cardoso, R. Majeska, K. Jepsen, M. Schaffler, Activation of Bone Remodeling after Fatigue: Differential Response to Linear Microcracks and Diffuse Damage, Bone. 47 (2010) 766-772. doi:10.3174/ajnr.A1256.Functional.

[16] O.D. Kennedy, M. Lendhey, P. Mauer, A. Philip, J. Basta-Pljakic, M.B. Schaffler, Microdamage induced by in vivo Reference Point Indentation in mice is repaired by osteocyte-apoptosis mediated remodeling, Bone. 95 (2017) 192-198. doi:10.1016/j.bone.2016.11.029.

[17] D. Vashishth, O. Verborgt, G. Divine, M. Schaffler, D. Fyhrie, Decline in osteocyte lacunar density in human cortical bone is associated with accumulation of microcracks with age, Bone. 26 (2000) 375-380.

[18] E. Radin, R. Rose, Role of subchondral bone in the initiation and progression of cartilage damage, Clin Orthop Relat Res. 213 (1986) 34-40.

[19] T. Funck-Brentano, H. Lin, E. Hay, M.D. Ah Kioon, C. Schiltz, D. Hannouche, R. Nizard, F. Lioté, P. Orcel, M.C. de Vernejoul, M.E. Cohen-Solal, Targeting bone alleviates osteoarthritis in osteopenic mice and modulates cartilage catabolism, PLoS One. 7 (2012). doi:10.1371/journal.pone.0033543.

[20] P. Sambrook, V. Naganathan, What is the relationship between osteoarthritis and ageing?, Baillieres. Clin. Rheumatol. 11 (1997) 695-710. doi:10.1016/S09503579(97)80004-0.

[21] J. Dequeker, J. Aerssens, F. Luyten, Osteoarthritis and osteoporosis: clinical and research evidence of inverse relationship, Aging Clin Exp Res. 15 (2003) 526-39.

[22] J. Pan, X. Zhou, W. Li, J.E. Novotny, S.B. Doty, L. Wang, In Situ measurement of transport between subchondral bone and articular cartilage, J Orthop Res. 27 (2009) 1347-1352. doi:10.1002/jor.20883.In.

[23] A. Kadri, H.K. Ea, C. Bazille, D. Hannouche, F. Lioté, M.E. Cohen-Solal, Osteoprotegerin inhibits cartilage degradation through an effect on trabecular bone in murine experimental osteoarthritis, Arthritis Rheum. 58 (2008) 2379-2386. doi:10.1002/art.23638.

[24] A. Kadri, T. Funck-Brentano, H. Lin, H.K. Ea, D. Hannouche, C. Marty, F. Lioté, V. Geoffroy, M.E. Cohen-Solal, Inhibition of bone resorption blunts osteoarthritis in mice with high bone remodelling, Ann. Rheum. Dis. 69 (2010) 1533-1538. doi:10.1136/ard.2009.124586.

[25] C. Sanchez, L. Pesesse, O. Gabay, J.P. Delcour, P. Msika, C. Baudouin, Y.E. Henrotin, Regulation of subchondral bone osteoblast metabolism by cyclic compression., Arthritis Rheum. 64 (2012) 1193-1203. doi:10.1002/art.33445.

[26] C. Sanchez, O. Gabay, C. Salvat, Y.E. Henrotin, F. Berenbaum, Mechanical loading highly increases IL-6 production and decreases OPG expression by osteoblasts, Osteoarthr. Cartil. 17 (2009) 473-481. doi:10.1016/j.joca.2008.09.007.

[27] S. Priam, C. Bougault, X. Houard, M. Gosset, C. Salvat, F. Berenbaum, C. Jacques, Identification of soluble $14-3-3 \varepsilon$ as a novel subchondral bone mediator involved in cartilage degradation in osteoarthritis, Arthritis Rheum. 65 (2013) 1831-1842. doi:10.1002/art.37951. 
[28] T. Funck-Brentano, W. Bouaziz, C. Marty, V. Geoffroy, E. Hay, M. Cohen-Solal, Dkk1-mediated inhibition of Wnt signaling in bone ameliorates osteoarthritis in mice, Arthritis Rheumatol. 66 (2014) 3028-3039. doi:10.1002/art.38799.

[29] W. Bouaziz, T. Funck-Brentano, H. Lin, C. Marty, H.K. Ea, E. Hay, M. Cohen-Solal, Loss of sclerostin promotes osteoarthritis in mice via $\beta$-catenin-dependent and independent Wnt pathways, Arthritis Res. Ther. 17 (2015) 24. doi:10.1186/s13075015-0540-6.

[30] N. Fazzalari, B. Vernon-Roberts, J. Carracott, Osteoarthritis of the hip: possible protective and causative roles of trabecular microfactures in the head of the femur, Clin Orthop Dis Rel Res. 216 (1983) 224-233.

[31] A. Jaiprakash, I. Prasadam, J.Q. Feng, Y. Liu, R. Crawford, Y. Xiao, Phenotypic Characterization of Osteoarthritic Osteocytes from the Sclerotic Zones : A Possible Pathological Role in Subchondral Bone Sclerosis, Int. J. Biol. Sci. (2012). doi:10.7150/ijbs.4221.

[32] D. Muratovic, D.M. Findlay, F.M. Cicuttini, A.E. Wluka, Y.-R. Lee, J.S. Kuliwaba, Bone matrix microdamage and vascular changes characterize bone marrow lesions in the subchondral bone of knee osteoarthritis, Bone. 108 (2018) 193-201. doi:10.1016/j.bone.2018.01.012.

[33] S. Touraine, H. Bouhadoun, K. Engelke, J.D. Laredo, C. Chappard, Influence of meniscus on cartilage and subchondral bone features of knees from older individuals: A cadaver study, PLoS One. 12 (2017) e0181956. doi:10.1371/journal.pone.0181956.

[34] D.B. Burr, M. Hooser, Alterations to the en bloc basic fuchsin staining protocol for the demonstration of microdamage produced in vivo, Bone. 17 (1995) 431-433. doi:10.1016/S8756-3282(95)00241-3.

[35] D.B. Burr, Stafford T., Validity of the bulk-staining technique to separate artifactual from in vivo bone microdamage, Clin. Orthop. Relat. Res. (1990) 305-308.

[36] K.P.H. Pritzker, S. Gay, S.A. Jimenez, K. Ostergaard, J.P. Pelletier, K. Revell, D. Salter, W.B. van den Berg, Osteoarthritis cartilage histopathology: Grading and staging, Osteoarthr. Cartil. 14 (2006) 13-29. doi:10.1016/j.joca.2005.07.014.

[37] G. Danilatos, Introduction to the ESEM instrument, Microsc Res Tech. 25 (1993) 35461.

[38] G. Danilatos, Bibliography of environmental scanning electron microscopy, Microsc Res Tech. 25 (1993) 529-34.

[39] P. Cabahug-Zuckerman, D. Frikha-Benayed, R.J. Majeska, A. Tuthill, S. Yakar, S. Judex, M.B. Schaffler, Osteocyte Apoptosis Caused by Hindlimb Unloading is Required to Trigger Osteocyte RANKL Production and Subsequent Resorption of Cortical and Trabecular Bone in Mice Femurs, J. Bone Miner. Res. 31 (2016) 13561365. doi:10.1002/jbmr.2807.

[40] W.Y. Cheung, J.C. Fritton, S.A. Morgan, Z. Seref-Ferlengez, J. Basta-Pljakic, M.M. Thi, S.O. Suadicani, D.C. Spray, R.J. Majeska, M.B. Schaffler, Pannexin-1 and P2X7Receptor Are Required for Apoptotic Osteocytes in Fatigued Bone to Trigger RANKL Production in Neighboring Bystander Osteocytes, J. Bone Miner. Res. 31 (2016) 890- 
899. doi:10.1002/jbmr.2740.

[41] J. Pan, B. Wang, W. Li, X. Zhou, T. Scherr, Y. Yang, C. Price, L. Wang, Elevated cross-talk between subchondral bone and cartilage in osteoarthritic joints, Bone. 51 (2012) 212-217. doi:10.1016/j.bone.2011.11.030.Elevated.

[42] T.R. Coughlin, O.D. Kennedy, The role of subchondral bone damage in post-traumatic osteoarthritis, Ann. N. Y. Acad. Sci. 1383 (2016) 58-66. doi:10.1111/nyas.13261.

[43] J.J. Stepan, D.B. Burr, I. Pavo, A. Sipos, D. Michalska, J. Li, A. Fahrleitner-pammer, H. Petto, M. Westmore, D. Michalsky, M. Sato, H. Dobnig, Low bone mineral density is associated with bone microdamage accumulation in postmenopausal women with osteoporosis, Bone. 41 (2007) 378-385. doi:10.1016/j.bone.2007.04.198.

[44] L. Cardoso, B.C. Herman, O. Verborgt, D. Laudier, R.J. Majeska, M.B. Schaffler, Osteocyte Apoptosis Controls Activation of Intracortical Resorption in Response to Bone Fatigue, J. Bone Miner. Res. 24 (2009) 597-605. doi:10.1359/jbmr.081210.

[45] A. Bertuglia, M. Lacourt, C. Girard, G. Beauchamp, H. Richard, S. Laverty, Osteoclasts are recruited to the subchondral bone in naturally occurring post-traumatic equine carpal osteoarthritis and may contribute to cartilage degradation, Osteoarthr. Cartil. 24 (2016) 555-566. doi:10.1016/j.joca.2015.10.008.

[46] T.M. Da Costa Gómez, J.G. Barrett, S.J. Sample, C.L. Radtke, V.L. Kalscheur, Y. Lu, M.D. Markel, E.M. Santschi, M.C. Scollay, P. Muir, Up-regulation of site-specific remodeling without accumulation of microcracking and loss of osteocytes, Bone. 37 (2005) 16-24. doi:10.1016/j.bone.2004.12.016.

[47] H. Jia, X. Ma, Y. Wei, W. Tong, R.J. Tower, A. Chandra, L. Wang, Z. Sun, Z. Yang, F. Badar, K. Zhang, W.J. Tseng, I. Kramer, M. Kneissel, Y. Xia, X.S. Liu, J.H.C. Wang, L. Han, M. Enomoto-Iwamoto, L. Qin, Loading-Induced Reduction in Sclerostin as a Mechanism of Subchondral Bone Plate Sclerosis in Mouse Knee Joints During LateStage Osteoarthritis, Arthritis Rheumatol. 70 (2018) 230-241. doi:10.1002/art.40351. 


\section{Figure legends}

\section{Figure 1: Representative images of microcracks}

(A-B) Hyaline cartilage (HC), calcified cartilage (CC) and microcracks (red arrow) detected by scanning electron microscopy with 500X magnification (A) or light microscopy with 10X magnification (B). (C) Dendrite network that abruptly stops at the border of calcified cartilage detected by epifluorescence microscopy with 20X magnification.

(D-E) Microcracks in the subchondral bone plate (SBP, red arrow) detected by scanning electron microscopy with $150 \mathrm{X}$ magnification (D) or light microscopy with $20 \mathrm{X}$ magnification (E).

(F) Density of osteocyte network in subchondral bone plate.

(G-I) Representative images of cartilage degradation with low damage (G), intermediate damage $(\mathrm{H})$ and severe cartilage damage $(\mathrm{I})$.

\section{Figure 2: Correlation between osteoarthritis (OA) score and microcracks}

(A) Correlation between OA score measured on each bone core with the Pritzker score and the density of microcracks in SBP and $\mathrm{CC}\left(\mathrm{Nb} / \mathrm{mm}^{2}\right)(\mathrm{r}=-0.447 \mathrm{p}=0.037)$ by Spearman test.

(B) Comparison of OA score between bone cores with no microcracks or with microcracks at both site. Data are mean $\pm \mathrm{SD} ;{ }^{*} \mathrm{p}<0.05$ by Mann-Whitney test.

(C) Effect of microcrack distribution effect on OA score. Data are presented using box plot; $* * \mathrm{p}<0.01$ by two way analysis of variance. 


\section{Figure 3: Microcracks and osteocyte density}

(A-B) Osteocyte density (Ot.Dn, No./mm2) by presence of microcracks in CC or SBP. Data are mean $\pm \mathrm{SD} ; * \mathrm{p}<0.05 * * \mathrm{p}<0.01$ by Mann-Whitney test.

(C) Correlation between volume/tissue volume (BV/TV, \%) and osteocyte density (Ot.Dn, No./mm2) at the SBP $(r=-0.470, p=0.045)$ calculated by Pearson test. $<0$, no microcracks; $\geq 1$, 1 or more microcracks.

Figure 4: Schematic view of the effect of microcracks on cartilage homeostasis and SBP osteocyte density

SBP microcracks are associated with less cartilage damage since absence of microcracks is linked to severe cartilage lesions. CC microcracks are associated with highest SBP osteocyte density and subchondral bone volume is inversely correlated with SBP osteocyte density.

\section{Supplemental Figure 1: Representation of the sampling site in the tibia plateau}

(A) Each bone core was taken from the media tibia plateau and was constantly removed at the same site (B) Representation of micro-CT acquisition from bone core. Red square represents the region of interest used for the quantification of bone parameters.

\section{Supplemental Figure 2: Correlation between bone parameters and cartilage degradation}

Correlation of histological OA score with (A) bone volume/tissue volume (BV/TV, \%) (r=0.31, $\mathrm{p}=0.12)$, (B) trabecular thickness (Tb.Th, $\mu \mathrm{m})(\mathrm{r}=-0.19, \mathrm{p}=0.24),(\mathrm{C})$ trabecular separation $(\mathrm{Tb} . \mathrm{Sp}, \mu \mathrm{m})(\mathrm{r}=-0.20, \mathrm{p}=0.23)$ and $(\mathrm{D})$ trabecular number $(\mathrm{Tb} . \mathrm{N}, 1 / \mathrm{mm})(\mathrm{r}=-0.31$, $\mathrm{p}=0.12$ ) calculated by Spearman test. 
Table: Subchondral bone parameters and microcracks in calcified cartilage and subchondral bone plate

Association of age (years), osteoarthritis (OA) score, bone volume/tissue volume (BV/TV, $\%)$, trabecular thickness $(\mathrm{Tb} . \mathrm{Th}, \mu \mathrm{m})$, trabecular separation (Tb. Sp, $\mu \mathrm{m})$, and trabecular number $(\mathrm{Tb} . \mathrm{N}, 1 / \mathrm{mm})$ by presence or absence of microcracks in calcified cartilage and/or subchondral bone plate. "No microcrack" or "Microcracks" indicate respectively absence or presence of microcracks both in the SBP and in the CC. The "CC group" or "SBP group" indicate the absence or the presence of microcracks at the considered site independently of the presence of microcracks in the other compartment. Data are mean \pm SEM. $* \mathrm{p}<0.05 * *$ $\mathrm{p}<0.01$ by Mann-Whitney test. $<0$, no microcracks; $\geq 1,1$ or more microcracks. 

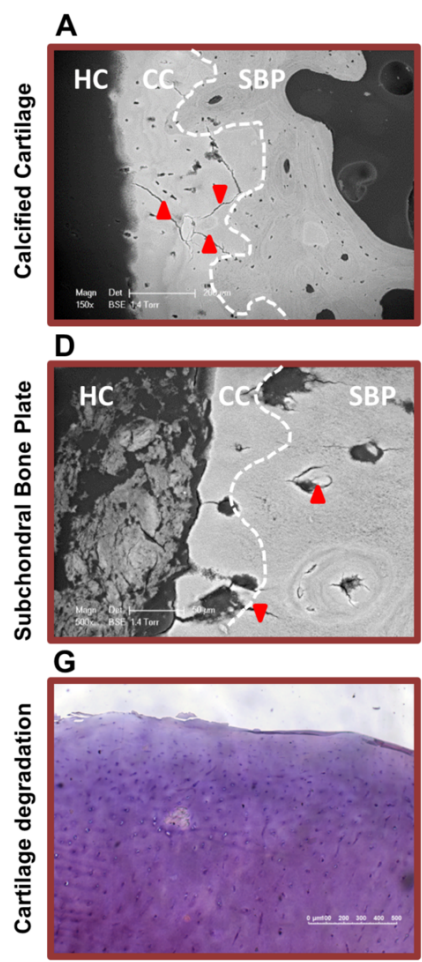

B

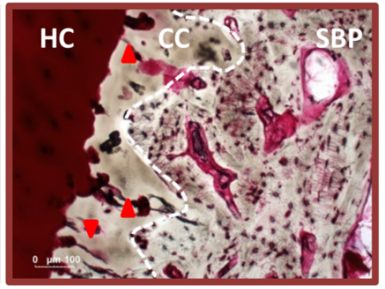

E

C

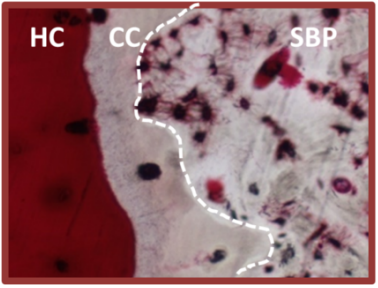

$F$
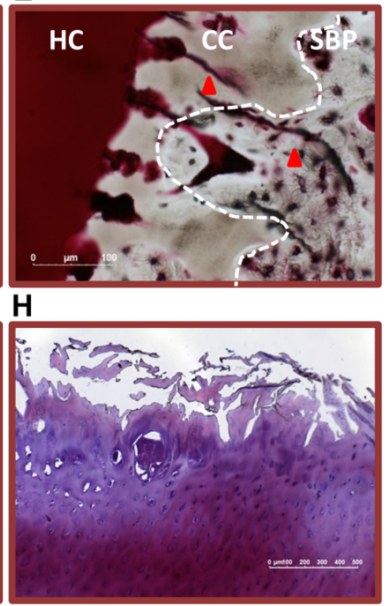

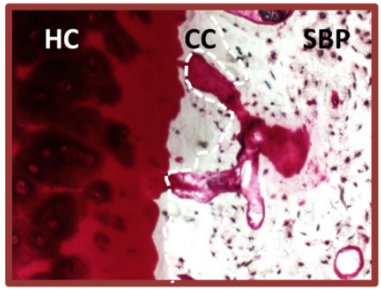

I

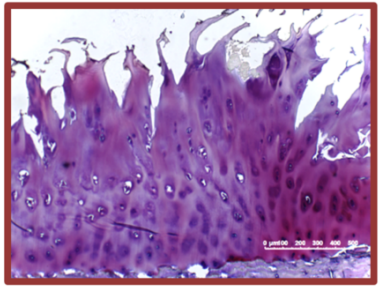


A

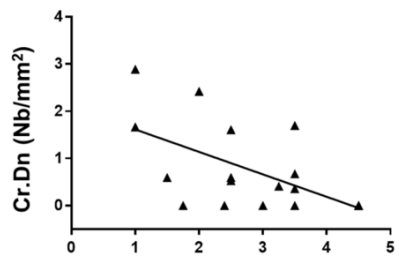

Histological OA score
B

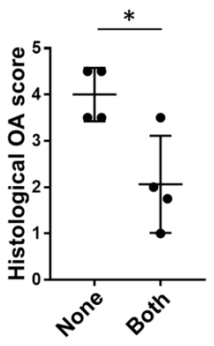

C

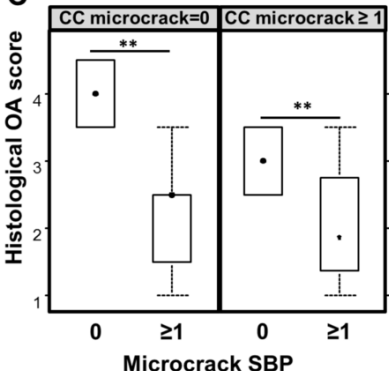




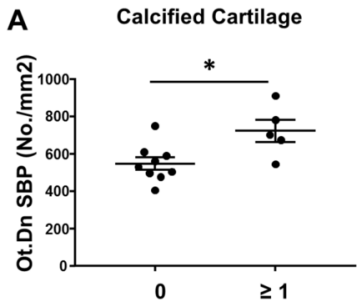

Microcracks
Subchondral Bone Plate

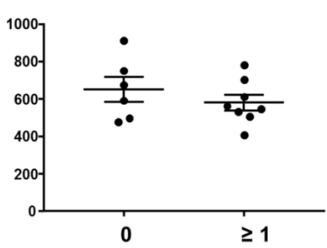

B

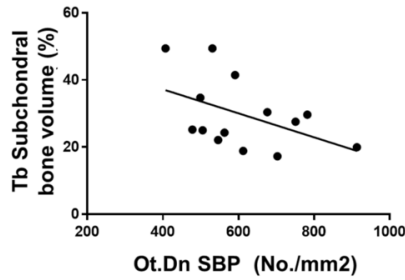




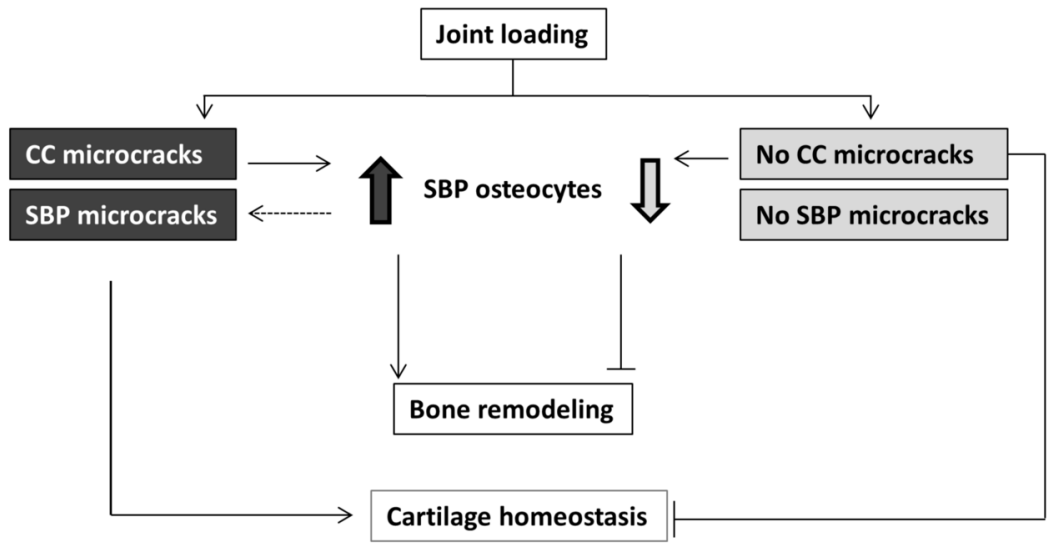




\begin{tabular}{|c|c|c|c|c|c|c|}
\hline & \multirow{2}{*}{$\begin{array}{c}\text { No microcrack } \\
0 \\
n=4\end{array}$} & \multirow{2}{*}{$\begin{array}{c}\text { Microcracks } \\
\geq 1 \\
n=4 \\
\end{array}$} & \multicolumn{2}{|c|}{ Calcified Cartilage } & \multicolumn{2}{|c|}{ Subchondral Bone Plate } \\
\hline & & & $\begin{array}{c}0 \\
n=9\end{array}$ & $\begin{array}{l}\geq 1 \\
n=6\end{array}$ & $\begin{array}{c}0 \\
n=6\end{array}$ & $\begin{array}{l}\geq 1 \\
n=9\end{array}$ \\
\hline Age (years) & $76.5 \pm 13.9$ & $79.3 \pm 11.2$ & $82.3 \pm 10.6$ & $81.7 \pm 11.6$ & $79.8 \pm 13.7$ & $83.6 \pm 8.6$ \\
\hline OA Score & $4.0 \pm 0.6$ & $2.1 \pm 1.0$ * & $3.0 \pm 1.2$ & $2.4 \pm 1.0$ & $3.7 \pm 0.8$ & $2.1 \pm 0.9^{\star *}$ \\
\hline Tb. BV/TV (\%) & $32.2 \pm 7.4$ & $26.3 \pm 8.3$ & $32.9 \pm 11.4$ & $25.9 \pm 7.2$ & $29.9 \pm 7.5$ & $30.2 \pm 12.2$ \\
\hline Tb.Th $(\mu \mathrm{m})$ & $157.0 \pm 26.0$ & $139.0 \pm 33.8$ & $154.9 \pm 34.4$ & $138.1 \pm 27.3$ & $150.0 \pm 24.0$ & $146.9 \pm 37.7$ \\
\hline Tb.Sp $(\mu \mathrm{m})$ & $383.3 \pm 12.7$ & $397.6 \pm 28.8$ & $370.7 \pm 68.8$ & $395.8 \pm 27.3$ & $386.3 \pm 18.9$ & $377.1 \pm 72.3$ \\
\hline Tb.N (1/mm) & $2.0 \pm 0.3$ & $1.9 \pm 0.4$ & $2.1 \pm 0.4$ & $1.9 \pm 0.3$ & $2.0 \pm 0.3$ & $2.0 \pm 0.5$ \\
\hline
\end{tabular}

